\title{
Finite element analysis for blood accumulation in intracerebral hemorrhage
}

\author{
PENG REN $^{1}$, BO-CHU WANG ${ }^{1}$, YA-ZHOU WANG ${ }^{1}$, HAI-JIAN XIA ${ }^{2}$, TING-WANG GUO ${ }^{1}$ and XIAO-FEI LI ${ }^{1}$ \\ ${ }^{1}$ Key Laboratory of Biorheological Science and Technology, Ministry of Education, College of Bioengineering, \\ Chongqing University, Chongqing 400044; ${ }^{2}$ Department of Neurosurgery, \\ The First Affiliated Hospital of Chongqing Medical University, Chongqing 400016, P.R. China
}

Received October 9, 2016; Accepted June 29, 2018

DOI: $10.3892 /$ etm. 2019.7474

\begin{abstract}
Biomechanical methods may provide a novel way to understand blood accumulation in intracerebral hemorrhage (ICH). The current study presents the results of a biomechanical analysis of blood accumulation in ICH using a finite element analysis, with an emphasis on the pressure exerted by the mass effect of blood in early ICH. A two-dimensional finite model of the human brain parenchyma and the human ventricular system was developed and analyzed under two preloading conditions. The material properties of the human parenchyma were derived from previous reports. Ogden's theory was applied to describe the stress-strain association in soft tissue. The results of the present study indicated that maximal stress was located at the two ends of the hemorrhage cavity, with the majority of stresses distributed on the zone surrounding the bleed. The two load environments demonstrated similar stress distributions. The loads put on the detached edges were not less than the intracranial pressure (ICP) when the stress threshold was reached. The results of the present study suggest that the direction of blood accumulation can be determined by the shape of the initial blood mass. Mechanical factors (blood pressure and ICP) did not serve a definitive role in preventing blood from accumulating in the early stages of ICH. The present study may aid in understanding the effects of mechanical factors in blood accumulation and hemostasis in patients with early $\mathrm{ICH}$.
\end{abstract}

\section{Introduction}

Intracerebral hemorrhage (ICH), a common stroke subtype with high morbidity and mortality, has become a topic of

Correspondence to: Professor Bo-Chu Wang, Key Laboratory of Biorheological Science and Technology, Ministry of Education, College of Bioengineering, Chongqing University, 174 Shazhengjie Street, Chongqing 400044, P.R. China

E-mail: wangbc2000@126.com

Key words: intracerebral hemorrhage, early onset, finite element analysis, stress distribution, blood accumulation increasing interest (1). The majority of reports on ICH have focused on the association between blood accumulation and mechanical factors, including blood pressure (BP), intracranial pressure (ICP) and hematoma pressure (2-5). However, there remain uncertainties regarding these factors, particularly in the early stages of ICH. The intracranial environment is a complex, multi-material composite system containing the skull (a rigid body), the parenchyma (between solid and liquid phases), cerebrospinal fluid (CSF; physical properties similar to water), blood (a highly viscous liquid) and blood vessels (elastic or hyperelastic solid). In a steady state, there are two main circulation systems: Blood and CSF; they are driven by pressure differentials that are markedly altered in patients with ICH. When ICH occurs, blood flows into the parenchyma and oppresses it, causing the steady state to break down and the pressure differentials to change correspondingly.

Considering the difficulty in directly measuring the changes in mechanical parameters caused by hemorrhagic sites, mathematical and physical analyses based on medical images are effective and convenient ways to investigate such parameters. Finite element analysis (FEA) is a commonly used method that simulates tissue deformation under a load. FEA provides a reliable method to evaluate the role that mechanical factors may serve, which may be otherwise difficult to test and verify. The present study aimed to demonstrate that hemostasis in ICH depends on biochemical rather than mechanical factors. It is clear, however, that mechanical changes in the course of cerebral hemorrhage precede biochemical changes. For this purpose, the mechanical properties of brain tissue, which undergoes large mechanical deformation induced by bleeding, should be given priority. The mechanical responses of brain tissue have attracted increasing attention over the past five decades (6-10). Numerous published studies (11-14) have investigated uniaxial compression and tension on brain tissues in various species, but only a few studies $(15,16)$ have utilized the human brain due to resource scarcity and ethical issues. However, a constitutive relationship, which is usually expressed by constitutive equation and can be broadly applicable in various fields, has not yet been obtained through previous studies. Existing studies that involved the modeling of the brain have used simplified models according to special demands, such as cerebrospinal fluid and brain dynamics (17) or hydrocephalus (18). The intracranial environment is complex and therefore requires a more complex 
model to accurately provide substantial amounts of data. In the present study, several assumptions were generated to simplify the complexity of the brain.

The present study investigated whether mechanical factors, particularly ICP and BP, serve an important role in inhibiting the accumulation of blood in ICH. An Ogden model based on the strain energy function was applied to describe the constitutive relationship. Geometric and material parameters were acquired from computed tomography (CT) images and tissue tension data, respectively, as previously described (15). Material parameters were then corrected by using experimental stress-strain data from a study by Jin et al (16). The published strain-stress association of human brain tissue was adopted to determine the deformation and load threshold (15). Different initial amounts for blood mass were included in the models as the contact edges. The results of stress distribution from the preloading conditions and different initial blood amounts were then compared. The results indicated that mechanical factors (BP and ICP) do not contribute to hemostasis in the early stages of $\mathrm{ICH}$.

\section{Materials and methods}

Assumptions. As previous studies have simple and specific models, a variety of assumptions were generated to simplify the complexity of the brain. Thus the present study produced a generalized model. The assumptions were as follows: i) The parenchyma was considered to be an isotropic, homogenous and incompressible material; ii) the skull was a boundary constraint, and CSF and blood masses were loading conditions; and iii) the model was formulated without time-dependent material behavior. In preloading case (PLC)1, no consideration was given to the physiological cyclical variation of ICP, thus ICP was set to a constant $1,300 \mathrm{~Pa}$ (as blood accumulates the corresponding CSF volume flows out of the system); and in the PLC2, no consideration was given to the physiological cyclical variation of ICP (ICP changed its value dynamically with the blood accumulation).

Geometry and meshing. The establishment of a digital model was performed in accordance with the study by Wittek et al (19). Patient geometric data for the parenchymal mesh were universal features obtained from preoperative CT data from the database of the First Affiliated Hospital of Chongqing Medical University (Chongqing, China). The model consisted of 4,313 8-node quadrilateral elements and 13,373 nodes, as shown in Fig. 1. The two hemispheres were analyzed, taking into account the possibility of midline migration caused by a jostle effect from a hematoma. Elements were detached along the maximum diameter of the blood mass on the cross-section and the position of the mass was referred to on CT images. The free edges were preset in the model to represent the contact edges between the blood mass and the parenchyma, with a range set between 7.407 and $43.682 \mathrm{~mm}$.

Material model for brain parenchyma. The hyperelastic Ogden model for strain energy was applied to explain the deformation of the brain parenchyma. The material behavior can be described by the Ogden hyperelastic formula:

$$
\mathrm{W}=\sum_{i=1}^{N} \frac{2 \mu_{i}}{\alpha_{i}{ }^{2}}\left(\lambda_{1}{ }^{\alpha_{i}}+\lambda_{2}{ }^{\alpha_{i}}+\lambda_{3}{ }^{\alpha_{i}}-3\right) .
$$

Where $\lambda_{i}$ are the principal stretch ratios, and $\mu_{i}$ and $\alpha_{i}$ are material coefficients, which are parameters determined by experimentation. It was assumed that the tissue kept a fixed volume and initial form during tension deformation. Thus,

$$
\lambda_{1} \lambda_{2} \lambda_{3}=1 \text { and } \lambda_{1}=\lambda_{T}, \lambda_{2}=\lambda_{3}=\lambda_{T}^{-\frac{1}{2}}
$$

Where $\lambda_{T}$ is the principal stretch ratio in the stretching direction. Then, in uniaxial tension,

$$
\mathrm{W}=\sum_{i=1}^{N} \frac{2 \mu_{i}}{\alpha_{i}^{2}}\left(\lambda_{T}^{\alpha_{i}}+2 \lambda_{T}^{-\frac{1}{2} \alpha_{i}}-3\right)
$$

The equation yields the following uniaxial tension stress component, $\sigma_{11}$, along the $\mathrm{x}_{1}$-axis:

$$
\sigma_{11}=\left(\lambda_{T}\right)=\sum_{i=1}^{N} \frac{2 \mu_{i}}{\alpha_{i}{ }^{2}}\left(\lambda_{T}^{\alpha_{i}-1}-\lambda_{T}{ }^{\frac{-1}{2} \alpha_{i}-1}\right) .
$$

For $\mathrm{N}=3$,

$$
\begin{aligned}
& \sigma_{11}=\mathrm{W}^{\prime}\left(\lambda_{T}\right)=\frac{2 \mu_{1}}{\alpha_{1}}\left(-\lambda_{T}^{-1-\frac{\alpha_{1}}{2}}+\lambda_{T}^{-1+\alpha_{1}}\right)+\frac{2 \mu_{2}}{\alpha_{2}} \\
& \left(-\lambda_{T}^{-1-\frac{\alpha_{2}}{2}}+\lambda_{T}^{-1+\alpha_{2}}\right)+\frac{2 \mu_{3}}{\alpha_{3}}\left(-\lambda_{T}^{-1-\frac{\alpha_{3}}{2}}+\lambda_{T}^{-1+\alpha_{3}}\right) .
\end{aligned}
$$

This equation can be utilized to calculate the required material parameters.

Boundary conditions. Given that the displacement of the outer brain surface is confined by the skull, all nodes were constrained on the outer edge with the exception of the gap edge between the left and right hemispheres, as shown in Fig. 1. In order to configure the terminal load condition, 4,676 Pa von Mises stress was adopted as the threshold, as previously shown (20), which was obtained through performing FEA on the data from Franceschini et al (15).

Loading. Two types of loads were applied in the simulations. First, a load was applied to the different lengths of the contact edge; this load was used to simulate the pressure applied on the edge by a blood mass in ICH. Two types of determined preloading conditions were applied on the ventricular edge as ICP. The brain model was analyzed using two distinct definitions of the prescribed nodal motion (referred to as PLC1 and PLC2). A stable load environment test (PLC1) was performed to estimate incremental pressure increases and establish a volume-pressure association. The nonlinear parameter estimation was achieved by the least square method using MATLAB 7.10 software (MathWorks, Inc., Natick, MA, USA). In the mutative load environment test (PLC2), the nonlinear FEA of blood accumulation was analyzed using Abaqus/Standards v. 6.12 software (Dassaut Systems, Waltham, MA, USA).

\section{Preloading}

PLC1. Using the normal ICP range (21), pressure was applied to the edge of the ventricles at 1,300 $\mathrm{Pa}$ to simulate ICP. A cavity in the parenchyma, which was formed by the simulated pressure, represented blood accumulation following parenchyma deformation. Applying the $\mathrm{ABC} / 2$ formula for $\mathrm{ICH}$ volume, the volume of the blood mass in the parenchyma was calculated using the 
A

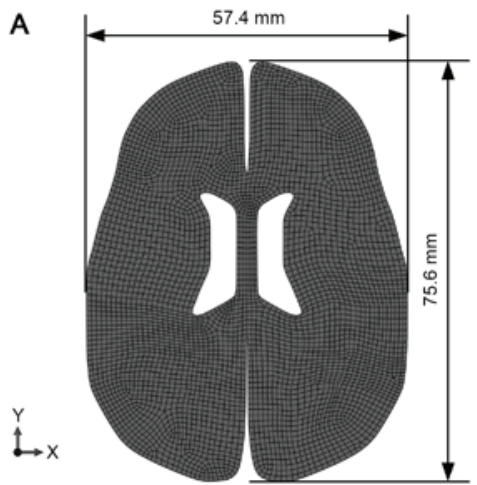

B

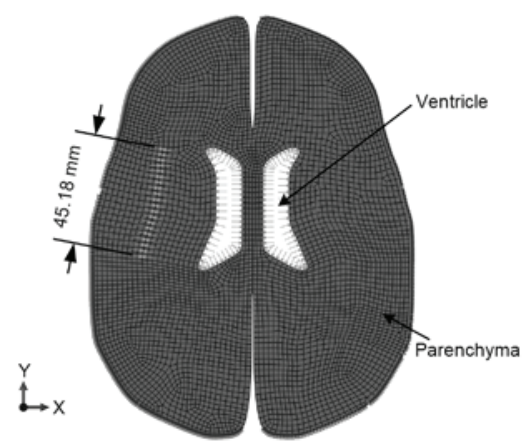

Figure 1. Model-produced parenchymal mesh. (A) An undeformed mesh and (B) a detached mesh representing the contact position between a blood mass and the parenchyma.

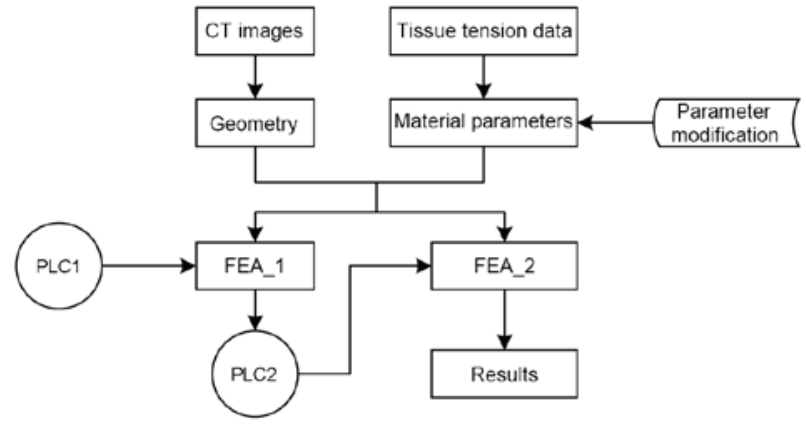

Figure 2. Schematic flow diagram of the methods used in the present study. $\mathrm{CT}$, computed tomography; PLC, preloading case; FEA, finite element analysis.

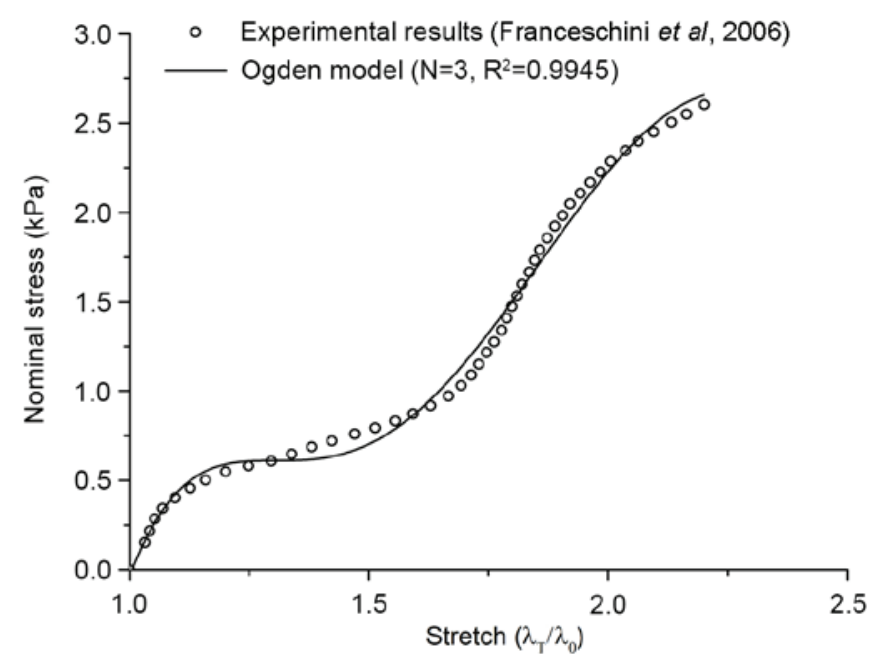

Figure 3. Data from the study by Franceschini et al (15) is a good fit with the Ogden model. $\lambda_{0}$, initial principal stretches. $\lambda_{\mathrm{T}}$, principal stretches at time $\mathrm{T}$.

following function: $V_{\text {ellipsoid }}=(\pi \times A \times B \times C) / 6$. Where $V_{\text {ellipsoid }}$ is the ellipsoid volume representing the blood mass volume, and $\mathrm{A}, \mathrm{B}$ and $\mathrm{C}$ represent the $\mathrm{X} / \mathrm{Y} / \mathrm{Z}$ axial dimensions of the ellipsoid in the Cartesian coordinate system, respectively. The length of the minor axis was set equal to the height of the ellipsoid.

PLC2. Similar to PLC1, pressure was exerted on the edge that was equal to the ICP. In the PLC2 simulation, the pressure
Table I. Coefficients defining the Ogden hyperelastic material for the parenchyma.

\begin{tabular}{lccc}
\hline & \multicolumn{3}{c}{ Factor } \\
\cline { 2 - 4 } $\mathrm{i}$ & $\mu_{\mathrm{i}}$ & $\alpha_{\mathrm{i}}$ & $\mathrm{D}_{1}$ \\
\hline 1 & -310.990 & -0.537 & \\
2 & 132.611 & -0.086 & 0.308 \\
3 & 180.541 & -1.032 & \\
\hline
\end{tabular}

$\mathrm{i}$, sequence number of material coefficients as subscript; $\mu_{\mathrm{i}}$ and $\alpha_{\mathrm{i}}$, Ogden's material coefficients of temperature dependence; $\mathrm{D}_{1}$, elastic material matrix.

increased with blood accumulation. As the CSF circulation was omitted, there were not enough conditions to apply a pressure-volume index method (22). Thus, using data acquired from the pressure-volume curve between ICP and incremental changes in intracranial content, as previously described (21), the pressure acting on the edge of the ventricles increased with increasing volume of blood in the skull.

Loading on the detached edge. Loads were applied to different lengths of detached edges, thus simulating hematoma pressure. The pressure was increased until a threshold stress occurred on the areas of partial stress concentration. The experimental procedure is demonstrated in Fig. 2.

\section{Results}

Material parameters. The Ogden model has previously been confirmed to be highly consistent with the results of Franceschini et al (15), as shown by the goodness of fit index (Fig. 3). The parameters for weighted material were obtained via examining the experimental data of Jin et al (16). The parameters for weighted material of the parenchyma are presented in Table I.

Finite element analyses. Following the application of a load under conditions described for PLC1, the volume of the cavity increased from 105 to $3,822 \mathrm{~mm}^{3}$ (data not shown). These 


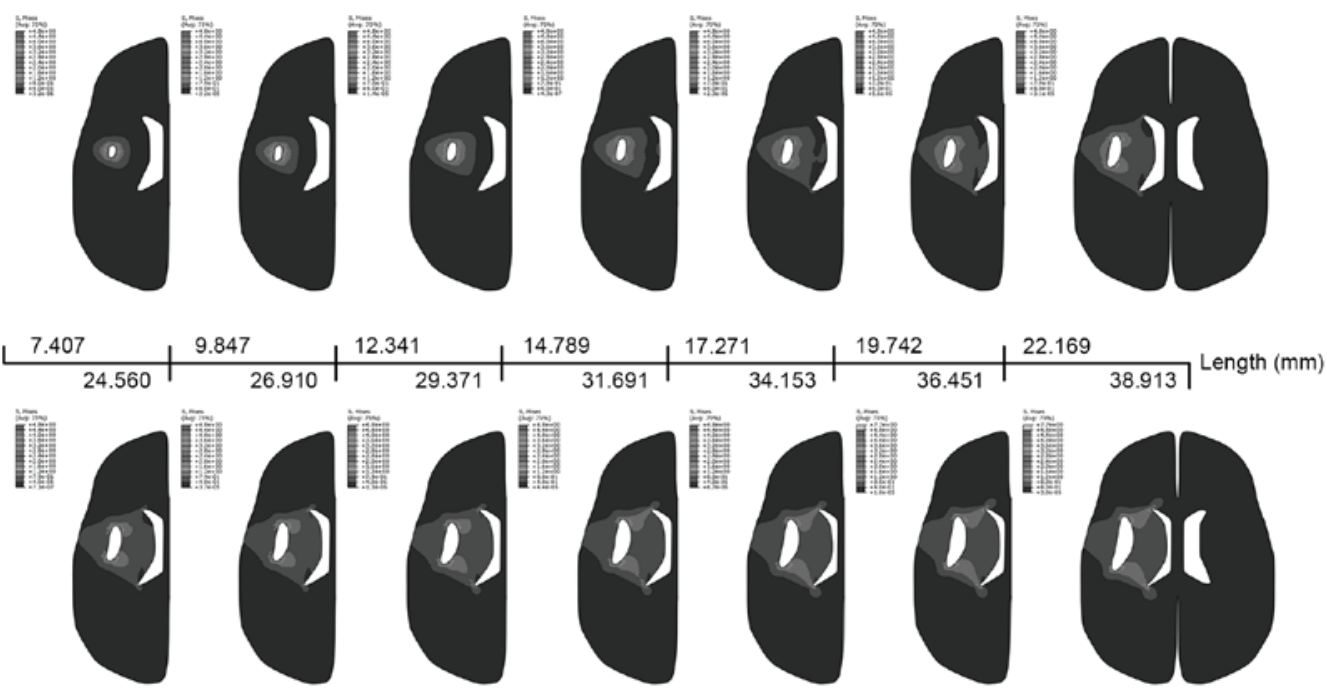

Figure 4. Finite element analyses of the von Mises stress distribution for preloading case 2 with different initial detached lengths.

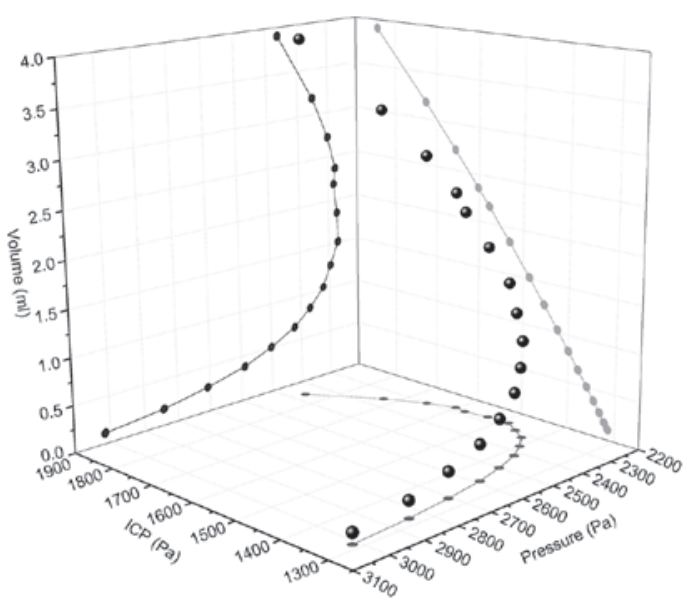

Figure 5. Association between blood mass pressure, ICP and cavity volume in preloading case 2 . ICP, intracranial pressure.

volume data were used to compute ICP incremental changes in PLC2 (data not shown). PLC2 simulated ICP increases with increasing contents in the skull, represented by the von Mises stress distribution in PLC2 (Fig. 4). Maximal stress was located at the two ends of the cavity, with the main stresses distributed in the surrounding zone. The ventricle began to be impacted when the length of the detached edge reached $19.741 \mathrm{~mm}$, but brain midline shift had not been observed in the simulations. A large level of stress was concentrated on the side of the ventricle when the length reached $36.451 \mathrm{~mm}$. The results from PLC1 demonstrated a similar stress distribution (data not shown).

Association between hematoma volume and pressure. Fig. 5 presents the association between blood mass pressure, blood mass volume and ICP in PLC2. With accumulating blood, the cavity volume of the parenchyma increased. The load demand for reaching a stress threshold on a stress concentrated position declined until ICP rapidly increased with cavity volume (from 2,180 to 3,907 $\mathrm{mm}^{3}$ ). Comparing the trends observed in PLC1 and PLC2 revealed that there was no distinct difference between the two preload cases if ICP was ignored (Fig. 6). Therefore, the

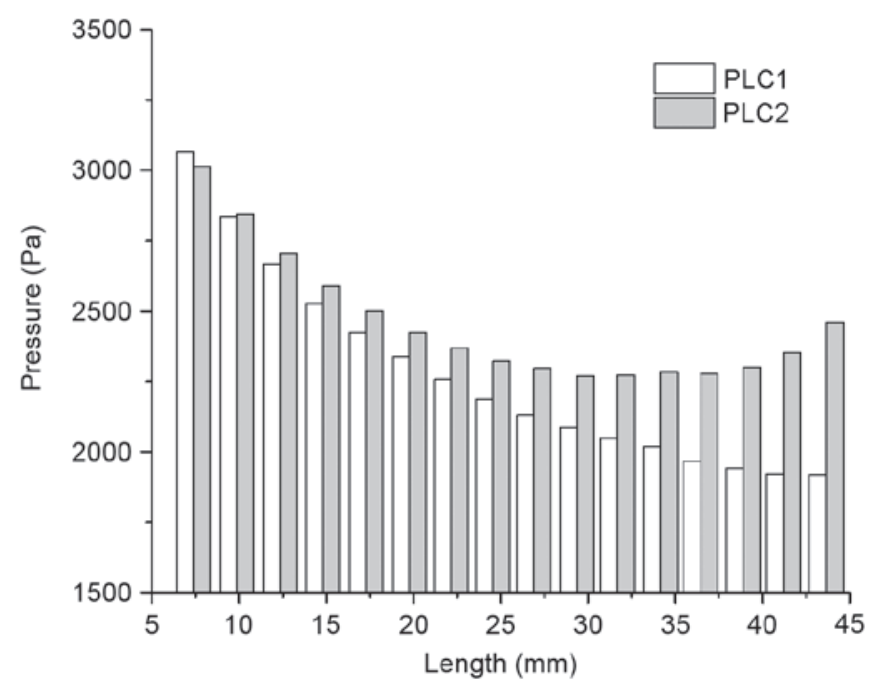

Figure 6. Final loads in PLC1 and PLC2 with different initial detached lengths. PLC, preloading case.

load, applied on the detached edges and resulting in destruction at the end of the crack, decreased with increasing detached length when the stress threshold was reached.

\section{Discussion}

During $\mathrm{ICH}$, blood accumulation in the parenchyma compresses surrounding tissues and eventually induces deformation. Discovering where stress may be distributed by this deformation may help predict locations of damage and the direction in which blood may continue to accumulate. The present study revealed that maximal stress occurred near the two ends of the detached edges of the blood mass in the direction of the major axis, indicating that the direction of blood accumulation was associated with the initial shape of the blood mass without consideration of anisotropy and inhomogeneity. The von Mises stress distribution in all the simulations was similar to hematoma geometry as observed on CT and magnetic resonance imaging (MRI). However, the volume 
of the blood mass in this simulation was far smaller than the volume calculated directly from CT images. Cells exposed to an abnormal mechanical environment may undergo apoptosis or necrocytosis (23-25). Under mechanical stress or stimulation, the physiological response of the cells may accelerate the spread of bleeding. The majority of medical images are generally acquired within $4 \mathrm{~h}$ of ICH onset, when erythrocyte lysis has not yet begun (26), but physiological changes in the tissue surrounding the blood mass are inevitable. The von Mises stress distribution may help predict the final shape of the hematoma as observed on CT and MRI images.

In $\mathrm{ICH}$, blood accumulates as a result of continued bleeding following vessel rupture. The reasons for blood to stop accumulating are typically considered to include biochemical factors and mechanical factors. Lowering blood pressure quickly following ICH so as to reduce blood accumulation is considered a potentially effective method to minimize $\mathrm{ICH}$-induced brain damage. However, there is no conclusive evidence to prove the efficacy of this strategy (1). Under normal physiological conditions, terminal arteriolar pressure is $\sim 40 \%$ of systemic arterial blood pressure (27). Intravascular pressure can be $\geq 6,500$ Pa under physiological conditions, even without considering patients with hypertension $(28,29)$. In the simulations performed in the present study, the pressure applied to the cavity edges increased continuously until a critical stress level was reached, with a maximum pressure value of 3,066 $\mathrm{Pa}$, which was still less than typical intravascular pressure. Thus, the stress caused by deformation in the parenchyma was not sufficient to stop blood from accumulating. Comparing the load change with the cavity edge between the two preloading conditions demonstrated that the critical pressure required for blood mass enlargement in the two cases decreased with increasing cavity volume. The change in the pressure had a similar trend when the weighted effect of ICP was not taken into consideration.

In conclusion, the results of the present study suggest that mechanical factors (BP and ICP) do not serve a decisive role in stopping blood from accumulating in the early stages of $\mathrm{ICH}$. Therefore, stress may be the primary contributing factor to the final shape of the hematoma in patients with $\mathrm{ICH}$.

\section{Acknowledgements}

The authors of the present study would like to acknowledge Professor Zhan-Fang Liu of the College of Aerospace Engineering at Chongqing University (Chongqing, China) for providing the Abaqus/Standards software.

\section{Funding}

The present study was supported by the National Basic Research 973 Program of China (grant no. 2014CB541600), the Visiting Scholar Foundation of Key Laboratory of Biorheological Science and Technology at Chongqing University (Chongqing, China), and the Ministry of Education (grant no. CQKLBST-2018-019).

\section{Availability of data and materials}

The datasets used and/or analyzed during the current study are available from the corresponding author on reasonable request.

\section{Authors' contributions}

PR performed the modeling, the finite element analysis and was a major contributor in writing the manuscript. BCW provided substantial contributions to the conception of the work. YZW and HJX provided the acquisition, analysis, and interpretation of data for the work. TWG and XFL performed the simulations and contributed in the preparation of the manuscript. All authors read and approved the final manuscript.

\section{Ethics approval and consent to participate}

Not applicable.

\section{Patient consent for publication}

Not applicable.

\section{Competing interests}

The authors declare that they have no competing interests.

\section{References}

1. Xi G, Strahle J, Hua Y and Keep RF: Progress in translational research on intracerebral hemorrhage: Is there an end in sight? Prog Neurobiol 115: 45-63, 2014.

2. Wu G, Xi G and Huang F: Spontaneous intracerebral hemorrhage in humans: Hematoma enlargement, clot lysis, and brain edema. Acta Neurochir Suppl 96: 78-80, 2006.

3. Steiner T, Al-Shahi Salman R, Beer R, Christensen H, Cordonnier C, Csiba L, Forsting M, Harnof S, Klijn CJ, Krieger D, et al: European stroke organisation (ESO) guidelines for the management of spontaneous intracerebral hemorrhage. Int J Stroke 9: 840-855, 2014

4. Kalita J, Misra UK, Vajpeyee A, Phadke RV, Handique A and Salwani V: Brain herniations in patients with intracerebral hemorrhage. Acta Neurol Scand 119: 254-260, 2009.

5. Hiploylee $\mathrm{C}$ and Colbourne $\mathrm{F}$ : Intracranial pressure measured in freely moving rats for days after intracerebral hemorrhage. Exp Neurol 255: 49-55, 2014.

6. Fung YCB: Elasticity of soft tissues in simple elongation. Am J Physiol 213: 1532-1544, 1967.

7. Zebian B and Critchley G: Spontaneous intracranial haemorrhage. Surgery (Oxford) 30: 136-141, 2012.

8. Estes MS and McElhane JH: Response of brain tissue to compressive loading. New York ASME, 1970.

9. Miller K and Chinzei K: Constitutive modelling of brain tissue: Experiment and theory. J Biomech 30: 1115-1121, 1997.

10. Goriely A, Geers MA, Holzapfel GA, Jayamohan J, Jérusalem A, Sivaloganathan S, Squier W, van Dommelen JA, Waters S and Kuhl E: Mechanics of the brain: Perspectives, challenges, and opportunities. Biomech Model Mechanobiol 14: 931-965, 2015.

11. Prevost TP, Balakrishnan A, Suresh S and Socrate S: Biomechanics of brain tissue. Acta Biomater 7: 83-95, 2011.

12. Bilston LE, Liu Z and Phan-Thien N: Large strain behaviour of brain tissue in shear: Some experimental data and differential constitutive model. Biorheology 38: 335-345, 2001.

13. Bayly PV, Black EE, Pedersen RC, Leister EP and Genin GM: In vivo imaging of rapid deformation and strain in an animal model of traumatic brain injury. J Biomech 39: 1086-1095, 2006.

14. Rashid B, Destrade M and Gilchrist MD: Mechanical characterization of brain tissue in compression at dynamic strain rates. J Mech Behav Biomed Mater 10: 23-38, 2012.

15. Franceschini G, Bigoni D, Regitnig P and Holzapfel GA: Brain tissue deforms similarly to filled elastomers and follows consolidation theory. J Mech Phys Solids 54: 2592-2620, 2006.

16. Jin X, Zhu F, Mao H, Shen M and Yang KH: A comprehensive experimental study on material properties of human brain tissue. J Biomech 46: 2795-2801, 2013. 
17. Linninger AA, Tangen K, Hsu CY and Frim D: Cerebrospinal fluid mechanics and its coupling to cerebrovascular dynamics. Ann Rev Fluid Mech 48: 219-257, 2016.

18. Taylor $\mathrm{Z}$ and Miller K: Reassessment of brain elasticity for analysis of biomechanisms of hydrocephalus. J Biomech 37 1263-1269, 2004.

19. Wittek A, Miller K, Kikinis R and Warfield SK: Patient-specific model of brain deformation: Application to medical image registration. J Biomech 40: 919-929, 2007.

20. Ren P, Wang BC, Wang YZ, Hao SL, Guo TW and Li XF. Evaluating tensile damage of brain tissue in intracerebral hemorrhage based on strain energy. Exp Ther Med 16: 4843-4852, 2018.

21. Marmarou A and Beaumont A: Physiology of the cerebrospinal fluid and intracranial pressure. In: Youmans neurological surgery. Winn HR (ed). 6th edition. Springer, Philadelphia, PA, pp169-182, 2011.

22. Maset AL, Marmarou A, Ward JD, Choi S, Lutz HA, Brooks D, Moulton RJ, DeSalles A, Muizelaar JP, Turner $\mathrm{H}$, et al: Pressure-volume index in head-injury. J Neurosurg 67: 832-840, 1987.
23. Tsai MS, Chou YL, Chang GL and Shen CL: The effect of magnitudes and duration of pressure on cerebral cortex in a rat model. J Clin Neurosci 8: 157-163, 2001.

24. Agar A, Li S, Agarwal N, Coroneo MT and Hill MA: Retinal ganglion cell line apoptosis induced by hydrostatic pressure. Brain Res 1086: 191-200, 2006.

25. Tök L, Nazıroğlu M, Uğuz AC and Tök O: Elevated hydrostatic pressures induce apoptosis and oxidative stress through mitochondrial membrane depolarization in PC12 neuronal cells: A cell culture model of glaucoma. J Recept Signal Transduct Res 34: 410-416, 2014.

26. Xi G, Keep RF and Hoff JT: Mechanisms of brain injury after intracerebral haemorrhage. Lancet Neurol 5: 53-63, 2006.

27. Gore RW: Pressures in cat mesenteric arterioles and capillaries during changes in systemic arterial blood pressure. Circ Res 34: 581-591, 1974.

28. Lipowsky HH: Microvascular rheology and hemodynamics. Microcirculation 12: 5-15, 2005

29. Boas DA, Jones SR, Devor A, Huppert TJ and Dale AM: A vascular anatomical network model of the spatio-temporal response to brain activation. Neuroimage 40: 1116-1129, 2008. 